\title{
Bone Marrow Endothelial Cells Induce Immature and Mature B Cell Egress in Response to Erythropoietin
}

\author{
Takeshi Ito $^{1,2}$, Yoko Hamazaki ${ }^{1,3^{*}}$, Akifumi Takaori-Kondo ${ }^{2}$, and Nagahiro Minato ${ }^{1}$ \\ ${ }^{1}$ Department of Immunology and Cell Biology, Graduate School of Medicine, Kyoto University, Sakyo-ku, \\ Kyoto 606-8501, Japan, ${ }^{2}$ Department of Hematology and Oncology, Graduate School of Medicine, Kyoto \\ University, Sakyo-ku, Kyoto 606-8507, Japan, ${ }^{3}$ Center for iPS Cell Research and Application (CiRA), \\ Laboratory of Immunobiology, Graduate School of Medicine, Kyoto University, Sakyo-ku, Kyoto 606-8501, \\ Japan
}

\begin{abstract}
Bone marrow stromal cells, including endothelial cells and mesenchymal stromal cells, support the maintenance, differentiation, and retention of hematopoietic stem and precursor cells under steady state conditions. At the onset of an emergency, such as severe blood loss or infection, the status of hematopoiesis in the bone marrow changes rapidly to ensure efficient production of cells of specific lineages; however, the function of stromal cells in emergency hematopoiesis has not been fully elucidated. Here, we unexpectedly found that B precursor, mature $B$, and $T$ cells were released from the bone marrow into the blood circulation in the early phase of hemorrhagic anemia and phenylhydrazine-induced hemolytic anemia. Administration of erythropoietin, which normally increases in response to anemia, stimulated the egress of $\operatorname{IgD}^{\text {low }}$ immature $B$ cells and recirculating mature B cells, which usually reside in the perivascular and intravascular space, from the bone marrow within 24 h. We also observed that endothelial cells in the bone marrow expressed erythropoietin receptor, and the expression levels were higher than those in other tissues. Erythropoietin stimulation of bone marrow endothelial cells induced the phosphorylation of STAT5 in vitro. Moreover, in vivo treatment with erythropoietin decreased surface VCAM1 expression and Cxcl12 transcription in bone marrow endothelial cells, both of which are essential for immature and mature $B$ cell retention in the bone marrow. These results suggest that bone marrow endothelial cells can sense and rapidly respond to erythropoietin increase during anemia, thereby regulating $B$ cell emigration from the bone marrow during emergency hematopoiesis.
\end{abstract}

Key words: erythropoietin, anemia, endothelial cells, B cell, bone marrow microenvironment

\section{Introduction}

Under steady state conditions, multilineage hematopoietic cells, including B cells, granulocytes, erythrocytes, and platelets, are produced in the bone marrow persistently throughout life. During emergency situations, such as bleeding or infection, the bone marrow rapidly produces massive numbers of specific lineages of hematopoietic cells, primarily in response to increased production of

\footnotetext{
*To whom correspondence should be addressed: Yoko Hamazaki, Department of Immunology and Cell Biology, Graduate School of Medicine, Kyoto University, Yoshida-konoe-cho, Sakyo-ku, Kyoto 606-8501, Japan.

Tel: +81-75-753-4433, Fax: +81-75-753-4403

E-mail: hamazaki@imm.med.kyoto-u.ac.jp

Abbreviations: CAR cell, Cxc112 abundant reticular cell; G-CSF, granulocyte colony stimulating factor; MSC, mesenchymal stromal cell; HSC, hematopoietic stem cell; MCP-1, monocyte chemotactic protein-1; LPS, lipopolysaccharide; PHZ, phenylhydrazine; VEGF, vascular endothelial growth factor.
}

hematopoietic cytokines. Acute anemia commonly occurs because of blood loss caused by severe trauma, various hemorrhagic disorders of the gastrointestinal tract, lung, and uterus, and, albeit less commonly, due to hemolytic anemia. A large amount of erythropoietin is produced in the kidneys immediately after the development of an anemic event to induce the proliferation and differentiation of erythroid progenitors (Jelkmann, 1992; Krantz, 1991).

Bone marrow microenvironments, including endothelial cells, mesenchymal stromal cells (MSC; including perivascular cells and Cxcl12 abundant reticular (CAR) cells), and osteoblasts, support hematopoiesis by producing cytokines and adhesion molecules under steady state conditions (Mendelson and Frenette, 2014; Morrison and Scadden, 2014). Recently, several reports have demonstrated that these bone marrow stromal cells rapidly respond to emergency situations to dynamically regulate hematopoiesis. During bacterial infection, endothelial cells stimulated by 
lipopolysaccharide (LPS) produce granulocyte colony stimulating factor (G-CSF) within $24 \mathrm{~h}$ and promote emergency granulopoiesis (Boettcher et al., 2014). MSCs also produce monocyte chemotactic protein-1 (MCP-1) in response to LPS to induce the emigration of monocytes from the bone marrow within $4 \mathrm{~h}$ (Shi et al., 2011). In addition, CAR cells decrease $\mathrm{B}$ cell trophic factors that indirectly respond to G-CSF and mediate the shift from lymphopoiesis to myelopoiesis (Day et al., 2015). These reports describe the response of bone marrow stromal cells to bacterial infection; however, how stromal cells behave during anemia, another hematologic emergency, has not been fully elucidated.

The location and emigration of developing hematopoietic stem and precursor cells in the bone marrow are also regulated by bone marrow stromal cells (Nagasawa, 2006). For example, hematopoietic stem cells (HSCs) reside near blood vasculature, where they interact with stem cell niche cells such as perivascular cells (Mendelson and Frenette, 2014; Morrison and Scadden, 2014). Immature B cells are retained in the perivascular parenchymal area and inside sinusoids to undergo negative selection before exiting the bone marrow (Batten and Osmond, 1984; Osmond and Batten, 1984; Pereira et al., 2009). Recirculating mature B cells traverse the sinusoidal vasculature to locate in the perivascular area, where they receive survival signals from perivascular dendritic cells (Cariappa et al., 2005; Sapoznikov et al., 2008). Their retention in the bone marrow is mediated by interactions between CXCR4 (a chemokine receptor) and CXCL12, and between alpha4 beta1 integrin (VLA-4) and VCAM1 (Koni et al., 2001; Leuker et al., 2001; Nie et al., 2004; Pereira et al., 2009). In this study, we unexpectedly found that immature and mature B cells in the bone marrow were released to the periphery immediately after the onset of anemia, and this release was mediated by the decreased expression of Vcam 1 and $\mathrm{Cxcl1} 2$ in bone marrow endothelial cells in response to erythropoietin. These findings suggest that bone marrow endothelial cells are able to respond to erythropoietin during the early phase of anemia and that the resulting functional alterations regulate emergency hematopoiesis.

\section{Materials and Methods}

\section{Mice}

Adult C57BL/6 mice of 8-12 weeks of age (Japan SLC) were maintained in standard pathogen-free conditions. To induce hemorrhagic anemia, mice were bled from the retro-orbital sinus to remove $400 \mu \mathrm{l}$ of blood under isoflurane anesthesia and intraperitoneally injected with $500 \mu \mathrm{l}$ PBS for volume replacement. Bleedings were performed for three consecutive days, and mice were analyzed on day 4. To induce hemolytic anemia, mice were intraperitoneally injected with $50 \mathrm{mg} / \mathrm{kg}$ phenylhydrazine (PHZ, Sigma Aldrich) for two consecutive days and analyzed on day 4 .
For erythropoietin treatment, mice were intraperitoneally injected with either $3000 \mathrm{U} / \mathrm{kg}$ recombinant human erythropoietin (rhEpo; Chugai, Epoetin beta) or PBS (control group) for the indicated periods of time. All experiments were performed according to the guidelines of the Animal Research Committee, Graduate School of Medicine, Kyoto University.

\section{Cell preparation from tissue samples}

Bone marrow cells were flushed out from femoral and tibial bones and suspended in media. To obtain endothelial cells and MSCs, bone marrow cells were incubated in D-MEM high glucose with $2 \%$ FCS, $5 \mathrm{mg} / \mathrm{ml}$ collagenase type I (Gibco), and $100 \mu \mathrm{g} / \mathrm{ml}$ DNase I (Worthington Biochemical Corporation) at $37^{\circ} \mathrm{C}$ for 20 min before cell suspension. To obtain endothelial cells, liver, spleen, lung, and kidney were dissected and incubated for $30 \mathrm{~min}$ before cell suspension in the media. To obtain osteoblasts, marrow depleted-bones were dissected and crushed and then incubated for $45 \mathrm{~min}$ before cell suspension in the media. The number of white blood cells and hematocrit in peripheral blood was measured using a Vetscan HM2 Analyzer (Abaxis).

\section{Flow cytometric analysis and sorting}

After lysing erythrocytes with ammonium-chloride-potassium (ACK) buffer, bone marrow, peripheral blood, and spleen cells were suspended in PBS with $2 \%$ FCS and 5 mM EDTA. After Fcblocking, samples were stained with antibodies (Supplemental Methods). Cells were analyzed using a FACS Canto II cytometer (BD biosciences), and data were analyzed with Flow Jo software (Flow Jo LLC).

To sort bone marrow or spleen endothelial cells and MSCs, collagenase digested bone marrow or spleen cells were Fc-blocked and stained with PE-conjugated anti-CD45 and anti-Ter119 and with biotin-conjugated anti-PDGFR $\beta$ antibodies. After washing, cells were incubated with anti-PE AutoMACS beads (Miltenyi Biotech), and the majority of hematopoietic cells were depleted using an AutoMACS Pro Separator (Miltenyi Biotech). Negatively collected cells were stained with FITC-conjugated antiCD31 and PECy7-conjugated anti-Scal antibodies and with APCconjugated streptavidin. Cell sorting was performed using a FACS Aria system (BD biosciences). To obtain osteoblasts, biotinconjugated CD51 antibody was used instead of anti-PDGFR $\beta$ antibody. To sort liver, lung, and kidney endothelial cells and hematopoietic cells from bone marrow, the AutoMACS depletion step was skipped.

\section{Immunostaining}

Femoral bones were fixed in 2\% paraformaldehyde for $2 \mathrm{~h}$ at room temperature and equilibrated in 30\% sucrose/PBS overnight. Fixed samples were embedded in SCEM (Section lab) and frozen in cold hexane. Sections were generated using the Kawamoto method (Kawamoto, 2003). Cryostat sections (6 $\mu$ m thick) were first blocked with 5\% normal donkey serum/PBS and then stained 
with antibodies (Supplemental methods). Sections were mounted with Mowiol (Sigma-Aldrich), and microscopic observation was performed using an Axiovert $200 \mathrm{M}$ inverted microscope (Zeiss).

\section{Quantitative RT-PCR analysis}

Sorted cells were lysed in Trizol reagent (Thermo Fisher Scientific). Following treatment with DNase I (Thermo Fisher Scientific), cDNA was synthesized using SuperScript III (Thermo Fisher Scientific) and Oligo-dT primers (Thermo Fisher Scientific) according to the manufacturer's instructions. cDNA was quantified by real-time PCR using a Light Cycler 480 II (Roche) and SYBR Green Master Mix (Roche). Expression levels of each gene were normalized to those of $A c t b$ in each sample. Primers are listed in supplemental methods.

\section{In vitro erythropoietin stimulation of endothelial cells}

Bone marrow cells were stained with biotinylated anti-VEGFR3, BV421-conjugated anti-CD45 and anti-Ter119, APC-conjugated anti-CD31, and PECy7-conjugated anti-Sca1 antibodies. After washing, bone marrow cells were incubated with anti-biotin AutoMACS beads and then positively sorted by AutoMACS. Cells were stimulated with $10 \mathrm{U} / \mathrm{ml} \mathrm{rhEpo} \mathrm{at} 37^{\circ} \mathrm{C}$ for $5 \mathrm{~min}$, prepared for Alexa 488-conjugated anti-pSTAT5 staining using Cytofix buffer and Perm Buffer III (BD Biosciences) according to the manufacturer's instructions, and analyzed using a FACS Canto II. Unstimulated samples were used as controls. To analyze erythroblasts and B cells in the bone marrow, the AutoMACS depletion step was skipped.

\section{Statistical analysis}

The significance of results was assessed using 2-tailed student ttests. $\mathrm{P}<0.05$ was considered significant. All data are presented means \pm S.E.M. ${ }^{*} \mathrm{P}<0.05$.

\section{Results}

\section{Hemorrhagic and hemolytic anemia induce rapid release of developing $B$ cells from the bone marrow to the periphery}

To assess the status of bone marrow hematopoiesis in the early phase of anemia, we induced hemorrhagic anemia in mice. Animals were bled for three consecutive days and analyzed on day 4 (Fig. 1A). Severe anemia was observed (Fig. S1A) and accompanied by elevated plasma erythropoietin concentration (Fig. S1B) and erythroblast proliferation in the bone marrow and spleen (Fig. S1C). The numbers of B cells at all stages of development, including pre-pro B cells, pro B cells, pre B cells, immature B cells, and mature $B$ cells, were significantly reduced in the bone marrow (Fig. 1B-C). Unexpectedly, IgD- immature B cells,
$\mathrm{IgD}^{\text {low }}$ immature B cells, and mature B cells were significantly increased in the spleen (Fig. 1D). The number of CD93 (C1qR1)-expressing B cells, which are recognized as $\mathrm{B}$ cells recently released from the bone marrow (Allende et al., 2010; Pereira et al., 2009), was significantly increased in both spleen and peripheral blood (Fig. 1D). We also found that the numbers of recirculating $\mathrm{T}$ cells and neutrophils in the bone marrow were reduced and that those in the spleen were increased (Fig. S1D). Similarly, in PHZ induced anemic mice (Fig. S1E-F), B cells, T cells, and neutrophils were significantly decreased in the bone marrow and increased in the spleen and peripheral blood (Fig. $1 \mathrm{E}-\mathrm{F}$ and Fig. S1G-H). These results suggest that hemorrhagic and hemolytic anemia rapidly induce the release of B-cell precursors, mature B cells, T cells, and neutrophils from the bone marrow to the periphery.

\section{Erythropoietin administration rapidly induces egress of immature and mature $B$ cells from the bone marrow to the periphery}

To examine the mechanism underlying the release of multiple lineages of cells from the bone marrow in the early phase of hemorrhagic and hemolytic anemia, we first analyzed the effect of erythropoietin, which is a major hematopoietic cytokine of erythroid lineage and is elevated in plasma during anemia (Jelkmann, 1992; Krantz, 1991) (Fig. 2A). $24 \mathrm{~h}$ after erythropoietin administration, basophilic and polychromatic erythroblasts were increased, whereas late stage orthochromatic erythroblasts were decreased in the bone marrow as observed in the anemia models (Fig. $\mathrm{S} 2 \mathrm{~A})$. The numbers of $\mathrm{IgD}^{-}$and $\mathrm{IgD}^{\text {low }}$ immature $\mathrm{B}$ cells and mature $\mathrm{B}$ cells in the bone marrow were significantly decreased (Fig. 2B-C), although the total number of cells in the bone marrow did not alter (Fig. S2B). Strikingly, the numbers of $\mathrm{IgD}^{\text {low }}$ immature $\mathrm{B}$ cells and mature $\mathrm{B}$ cells in the bone marrow decreased by $60 \%$ and $90 \%$, respectively (Fig. 2C). In peripheral blood, the total numbers of white blood cells, $\mathrm{IgD}^{-}$and $\mathrm{IgD}^{\text {low }}$ immature $\mathrm{B}$ cells, and mature $\mathrm{B}$ cells were significantly increased (Fig. 2D), as were those of $\mathrm{CD}^{+}$B cells (Fig. 2D). A trend towards increased numbers of immature $B$ cells in the spleen was also observed (Fig. 2E). The decreased numbers of other developing $\mathrm{B}$ cell populations and neutrophils detected in the bone marrow of the anemia models were not observed at least $24 \mathrm{~h}$ after erythropoietin treatment (Fig. 2C and Fig. $\mathrm{S} 2 \mathrm{C}$ ). $\mathrm{CD}^{+}$and $\mathrm{CD}^{+} \mathrm{T}$ cells were also significantly reduced in the bone marrow and tended to increase in the peripheral blood and spleen (Fig. S2C). The number of HSCs was slightly decreased in the bone marrow, and increased in the spleen (Fig. S2C). Consistent with prior reports (Cariappa et al., 2005; Pereira et al., 2009), immunohistochemical staining indicated that $\operatorname{IgD}^{+} \mathrm{B}$ cells resided both inside $\mathrm{VEGFR}^{+}$sinus vasculature and in the perivascular area. After administration of erythropoietin, $\mathrm{IgD}^{+}$ 
A

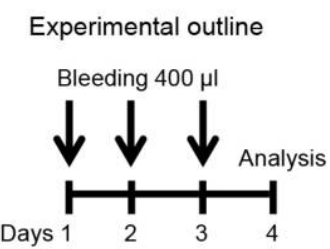

B

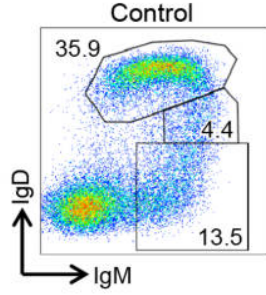

Bleeding

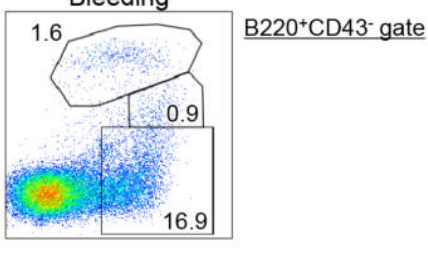

C

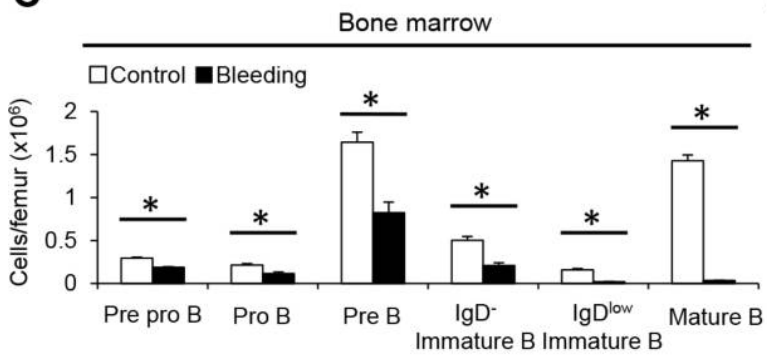

E

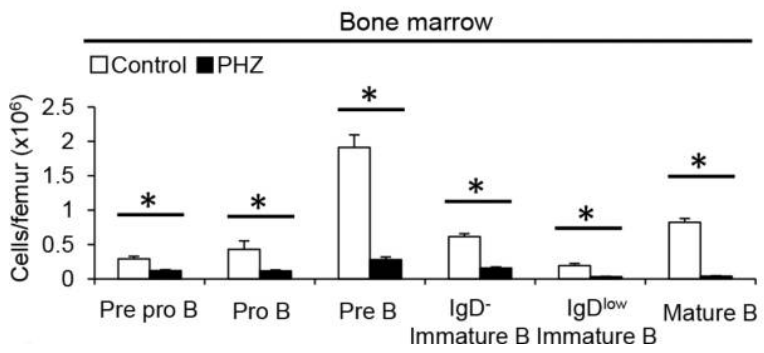

D

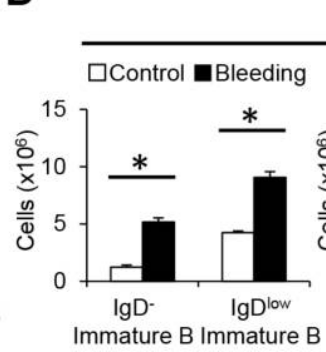

Spleen

PB
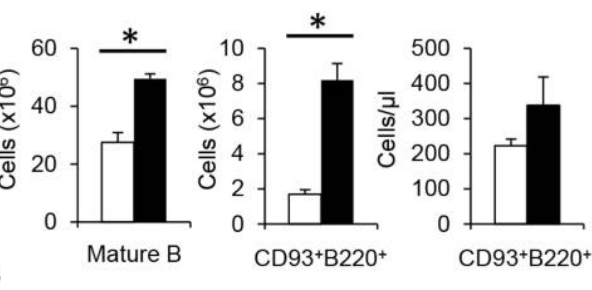

$\mathbf{F}$

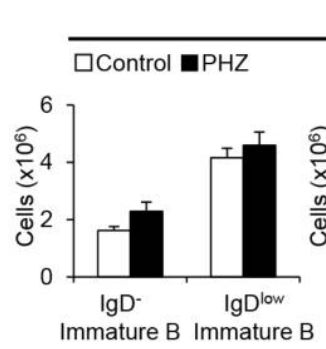

Spleen

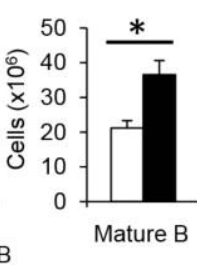

PB
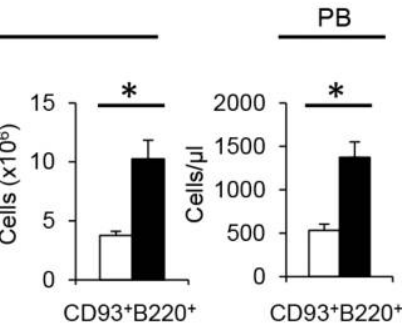

Fig. 1. Hemorrhagic and hemolytic anemia induce rapid release of developing B cells from the bone marrow to the periphery. (A) Experimental outline. Mice were bled to remove $400 \mu \mathrm{l}$ of blood for three consecutive days and analyzed on day 4 . (B) Representative FACS plot of bone marrow B220 $0^{+} \mathrm{CD} 43$ $\mathrm{B}$ cells in control and bled mice. The gates show mature B cells (top, $\operatorname{IgD}^{+} \operatorname{IgM}^{+}$), $\operatorname{IgD}^{\text {low }}$ immature $\mathrm{B}$ cells (middle, $\operatorname{IgD}^{\text {low }} \operatorname{IgM}{ }^{+}$), and IgD immature $\mathrm{B}^{-}$cells (bottom, $\operatorname{IgD}^{-} \operatorname{IgM}^{+}$). (C) Numbers of pre-pro B, pro B, pre B, $\operatorname{IgD}^{-}$immature $\mathrm{B}$, IgD ${ }^{\text {low }}$ immature $\mathrm{B}$, and mature B cells per femur in control and bled mice

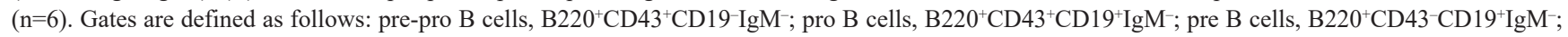

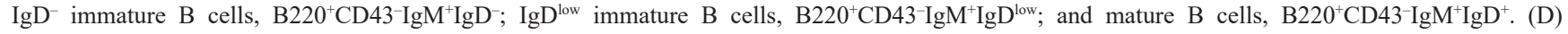
Numbers of $\mathrm{IgD}^{-}$immature $\mathrm{B}$, IgD $\mathrm{I}^{\text {low }}$ immature $\mathrm{B}$, mature $\mathrm{B}$, and $\mathrm{CD} 93^{+} \mathrm{B} 220^{+} \mathrm{B}$ cells in the spleen $(\mathrm{n}=6)$ and $\mathrm{CD} 93^{+} \mathrm{B} 220^{+} \mathrm{B}$ cells in the peripheral blood (PB) $(n=6)$. (E) Numbers of $B$ cells at each stage of development per femur in control and PHZ-induced anemic mice ( $\mathrm{n}=7$ ). (F) Numbers of IgD immature B, IgD low immature B, mature B, and CD93 ${ }^{+} \mathrm{B} 220^{+} \mathrm{B}$ cells in the spleen $(\mathrm{n}=5-7)$, and $\mathrm{CD} 93^{+} \mathrm{B} 220^{+} \mathrm{B}$ cells in the $\mathrm{PB}(\mathrm{n}=5)$.

B cells almost disappeared from the bone marrow, whereas the distribution of $\mathrm{Gr}-1^{+}$neutrophils remained unchanged (Fig. 2F). These results indicate that erythropoietin rapidly promotes the release of newly formed immature B cells and recirculating mature $\mathrm{B}$ cells and $\mathrm{T}$ cells from the bone marrow.

\section{Bone marrow endothelial cells express erythropoietin receptor}

We sought to identify cell subsets that expressed the erythropoietin receptor, which would render sensitivity to erythropoietin. We sorted various subsets of hematopoietic and non-hematopoietic cells and examined their expression of erythropoietin receptor transcripts (Epor). Among hematopoietic lineages, Epor was highly expressed in erythro- blasts, as expected, but was almost undetectable in B and $\mathrm{T}$ cells (Fig. 3A). Strikingly, Epor was also expressed in bone marrow endothelial cells at approximately $40 \%$ of the level observed in erythroblasts (Fig. 3A and Fig. S3). Bone marrow endothelial cells were defined as CD45-Ter119 $\mathrm{CD}_{31}{ }^{+} \mathrm{Sca}^{+}$, and their expression of endothelial cell markers including Tie-2, VE-cadherin, VEGFR-2, and VEGFR-3, was confirmed (Fig. S3). MSCs and macrophages (Chow et al., 2011) expressed low but detectable levels of Epor (Fig. 3A and Fig. S3). Furthermore, Epor expression in bone marrow endothelial cells was significantly higher than that in endothelial cells of other organs (Fig. 3B).

To evaluate whether or not erythropoietin receptor is functional in bone marrow endothelial cells, we directly stimulated bone marrow endothelial cells with erythropoie- 
A

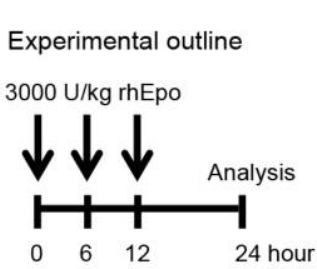

B
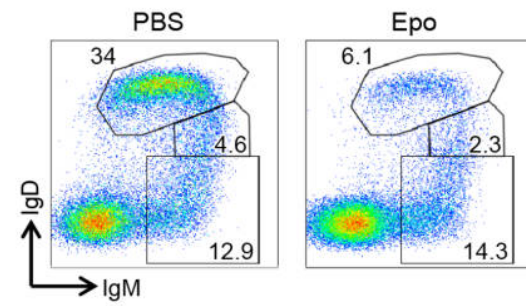

B220+CD43- gate

D

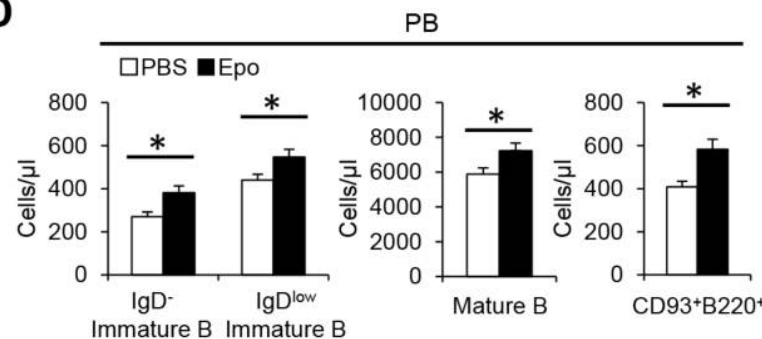

$\mathbf{E}$

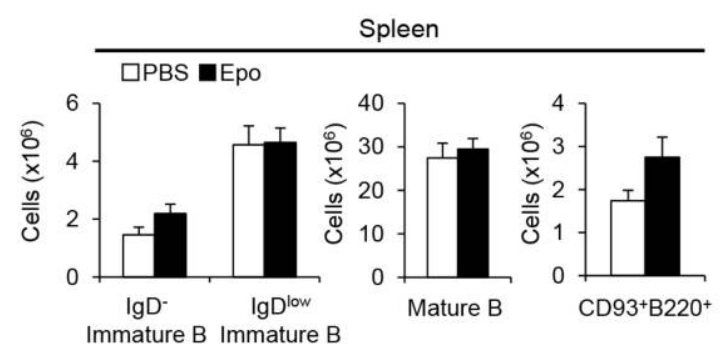

$\mathbf{F}$

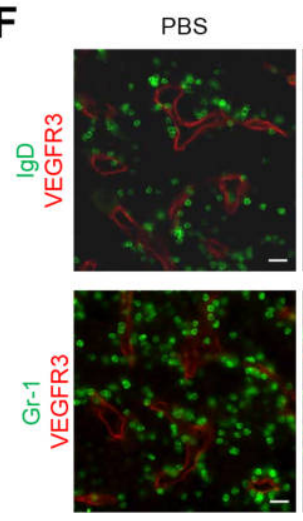

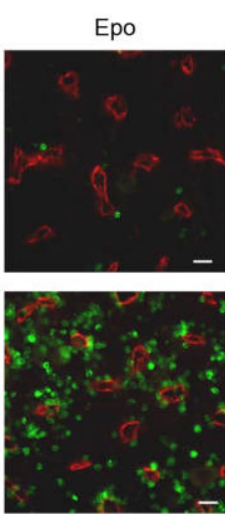

Fig. 2. Erythropoietin administration rapidly induces egress of immature and mature B cells from the bone marrow to the periphery. (A) Experimental outline. Recombinant human erythropoietin (Epo) was injected three times, and samples were analyzed $24 \mathrm{~h}$ after the first injection. (B) Representative FACS plot of bone marrow B220 $0^{+} \mathrm{CD} 43^{-} \mathrm{B}$ cells in PBS or Epo treated mice. (C) Numbers of B cells at different stages during development per femur in PBS or Epo treated mice $(\mathrm{n}=6)$. (D-E) Numbers of $\mathrm{IgD}^{-}$immature $\mathrm{B}, \mathrm{IgD}^{\text {low }}$ immature $\mathrm{B}$, mature $\mathrm{B}$, and $\mathrm{CD} 93^{+} \mathrm{B} 220^{+} \mathrm{B}$ cells in the PB $(\mathrm{D})$ and spleen $(\mathrm{E})$ in PBS- or Epo-treated mice (PB, $n=12$; spleen, $n=6)(F)$ (Left) Immunostaining of bone marrow samples from PBS- or Epo-treated mice stained with IgD

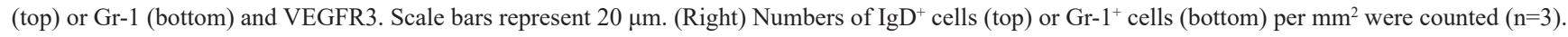

tin for 5 min in vitro. Erythropoietin stimulation of bone marrow endothelial cells induced the phosphorylation of STAT5, a major downstream target of erythropoietin receptor, as well as erythroblasts (Fig. 3C). In contrast, the phosphorylation of STAT5 was not observed in B cells (Fig. $3 \mathrm{C})$. These results indicate that bone marrow endothelial cells express functional erythropoietin receptor and can potentially respond to erythropoietin.

\section{Erythropoietin treatment led to decreased Cxcl12 expression in bone marrow endothelial cells}

We next examined the effect of erythropoietin administration on chemokine and cytokine expression in bone marrow endothelial cells. Bone marrow endothelial cells were sorted $48 \mathrm{~h}$ after erythropoietin treatment, and the levels of various transcripts were evaluated. We observed that Cxcl12 and Il7 expression levels were significantly decreased, whereas those of Kitl (Stem cell factor), Flt3l, Jag1 (Jagged-1), and Sele (E-selectin) were unchanged (Fig. 4 and Fig. S4A). In contrast, Il6 and Mmp9 (Matrix Metalloproteinase) expression was significantly increased (Fig. 4 and Fig. S4A). The reduction of Cxcll2 and Il7 mRNA levels was also observed in MSCs; however, the difference was less significant than that observed in endothelial cells (Fig. S4B). These results indicate that erythropoietin immediately alters the expression of various hematopoietic factors, including chemokines and cytokines essential for B cell retention and development in bone marrow endothelial cells. 
A

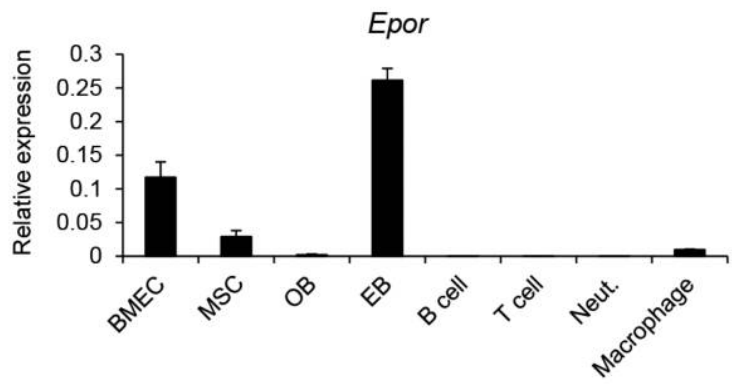

B

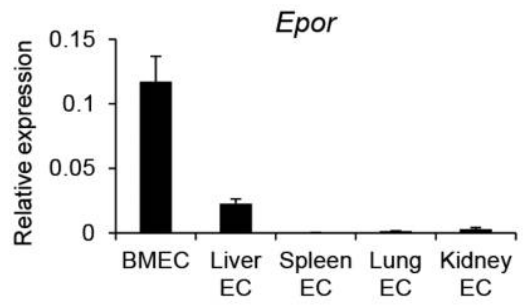

C

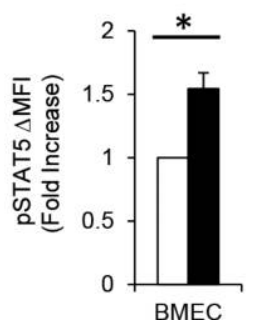

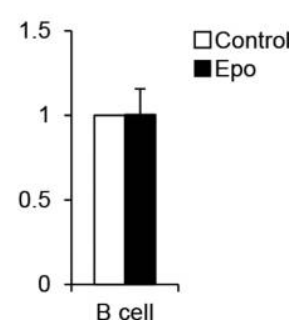

Fig. 3. Bone marrow endothelial cells express erythropoietin receptor. (A) Epor mRNA expression relative to that of Actb in the indicated cell populations. Abbreviations are as follows: BMEC, bone marrow endothelial cells; OB, osteoblasts; EB, erythroblasts; Neut., neutrophils. Sorting gates were defined as follows: BMECs, CD45-Ter119 $\mathrm{CD} 1^{+} \mathrm{Sca}^{+}$; MSCs, CD45-Ter119-PDGFR $\beta^{+} \mathrm{CD} 31^{-}{ }^{-} \mathrm{Sca} 1^{-}$; OBs, CD45 Ter119- ${ }^{-}$D51 $1^{+} \mathrm{CD}^{-} 1^{-} \mathrm{Sca} 1^{-}$; EBs, CD71 ${ }^{+}$Ter119 ${ }^{+}$; B cells, B220 ${ }^{+}$; $\mathrm{T}$ cells, $\mathrm{CD}^{+}$; Neut., CD $11 \mathrm{~b}^{+} \mathrm{Gr}-1^{+}$; Macrophages, Gr- ${ }^{-} \mathrm{CD} 115^{-} \mathrm{F} 4 / 80^{+} \mathrm{FSC}^{\text {low }}$. $(\mathrm{n}=3-4)$ (B) Epor mRNA expression in endothelial cells from the indicated organs relative to Actb $(\mathrm{n}=3)$. Endothelial cells in each organ were defined as $\mathrm{CD} 45^{-} \mathrm{Ter} 119^{-} \mathrm{CD} 31^{+} \mathrm{Sca} 1^{+}$. (C) Phosphorylation of STAT5 in BMECs, EBs, and B cells stimulated with Epo (10 U/ml) for 5 min in vitro was evaluated by FACS. Fold increase of $\Delta$ mean fluorescence intensity $(\triangle \mathrm{MFI})$ in Epo stimulated samples relative to that in unstimulated samples is shown. Data are average of three independent experiments.

\section{Erythropoietin treatment led to decreased VCAM1 expression in bone marrow endothelial cells}

In addition to CXCL12, the expression of VCAM1 in endothelial cells is essential for immature and mature B cell retention in the bone marrow (Koni et al., 2001). FACS analysis revealed that surface VCAM1 expression in endothelial cells was significantly decreased, with a $70 \%$ reduction in mean fluorescence intensity $24 \mathrm{~h}$ after the injection of erythropoietin (Fig. 5A-B). In contrast, the expression of ICAM-1, another important adhesion molecule, was not affected (Fig. 5A). The changes in VCAM1 expression in MSCs and macrophages were less significant than those observed in endothelial cells (Fig. 5A-B). Although VCAM1 can be cleaved by proteolytic enzymes (Levesque et al., 2001), reduced Vcam1 expression was observed at the mRNA level after erythropoietin administration (Fig. 5C). We also observed that VCAM1 expression in endothelial cells was significantly decreased under anemic conditions (Fig. 5D). These data suggest that endothelial cells can rapidly sense and respond to increases in plasma erythropoietin in acute anemia, leading to decreases in their expression of Cxcl12 and Vcaml and the rapid emigration of immature and mature B cells from the bone marrow (Fig. S5).

\section{Discussion}

In this study, we have demonstrated that immature and mature $\mathrm{B}$ cells rapidly egress from the bone marrow to the periphery in early phase of anemia. Furthermore, we show this egress is caused by an immediate response to erythropoietin produced by bone marrow stromal cells, mainly endothelial cells, which leads to a decrease of $\mathrm{Cxcl12}$ and Vcam 1 expression. Hence, our study reveals a novel function of erythropoietin on bone marrow endothelial cells in emergency hematopoiesis.

Both the location of immature B cells within the bone marrow during development and the entry and egress of recirculating B cells are tightly regulated (Cariappa et al., 2005; Nagasawa, 2006; Pereira et al., 2009). The interaction between integrin VLA-4 and VCAM1, particularly in bone marrow endothelial cells, is crucial for the retention of immature and mature B and T cells (Koni et al., 2001; Leuker et al., 2001; Pereira et al., 2009). The interaction between the chemokine receptor CXCR4 and the chemokine CXCL12, which can activate VLA-4 (Glodek et al., 2003), is also indispensable (Allende et al., 2010; Martin et al., 2003; Nie et al., 2004). The immediate decrease of Vcam 1 and $\mathrm{Cxcl12}$ expression in bone marrow endothelial cells and the egress of immature and mature B cells induced by erythropoietin clearly occurred in the absence of an increase in total bone marrow cell number, suggesting the involvement of specific molecular interactions in B cell export. Furthermore, B cells neither expressed Epor nor phosphorylated STAT5 in response to erythropoietin. Thus, although we do not have direct evidence, our data strongly suggest that decreased levels of VCAM1 and CXCL12 in bone marrow endothelial cells are responsible for the rapid emigration of immature and mature B cells from the bone marrow. Consistently, T cells, which recirculate into the bone marrow by similar molecular interactions, also emigrated to the periphery (Koni et al., 2001; Mazo et al., 2005). At the same time, there remains a possibility that another mechanism for B cell retention in the bone marrow, 


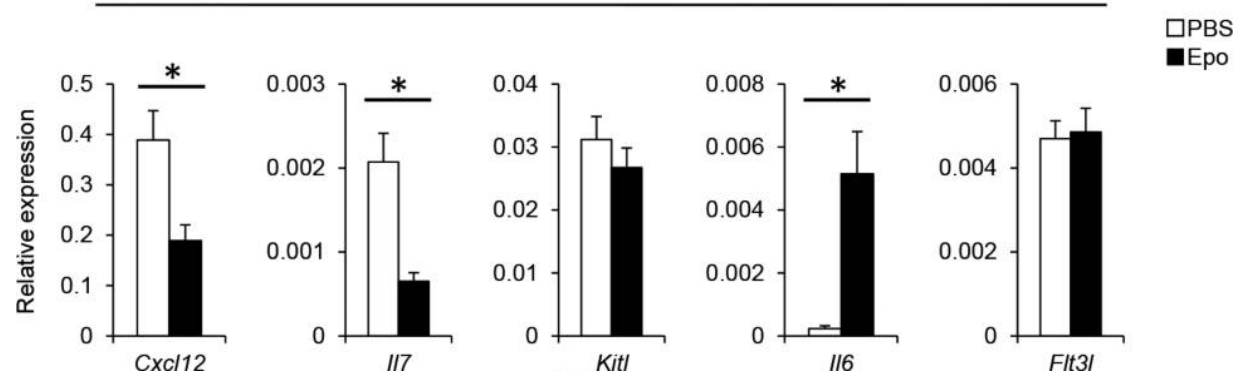

Fig. 4. Erythropoietin treatment leads to decreased $\mathrm{Cxcl12}$ expression in bone marrow endothelial cells. Expression levels of the indicated genes relative to that of Actb in sorted BMECs from PBS- or Epo-treated mice. Mice were treated with PBS or Epo (3000 U/kg) once daily for two consecutive days, and cells were sorted on day $3(n=8)$.

A

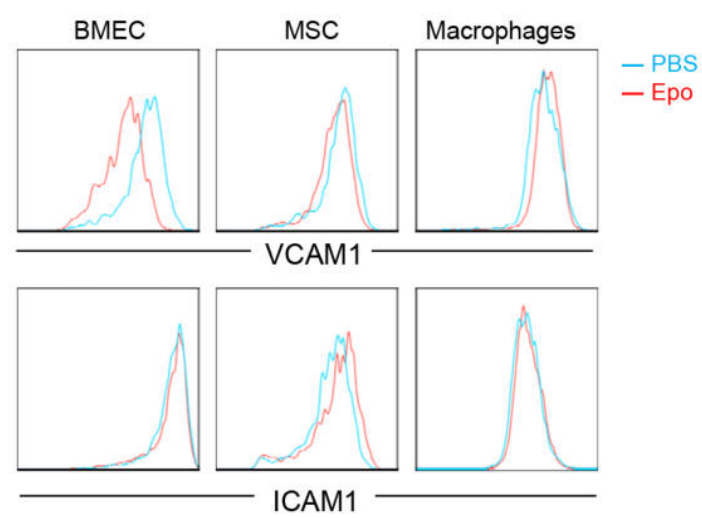

C

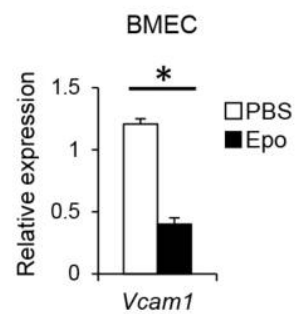

B

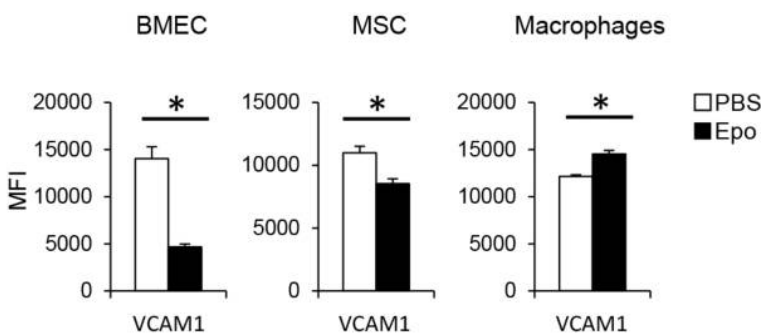

Fig. 5. Erythropoietin decreased VCAM1 expression in bone marrow endothelial cells. (A) Representative FACS histograms of VCAM1 (top) and ICAM1 (bottom) in BMECs, MSCs, and macrophages in PBS- (blue line) and Epo- (red line) treated mice. The experimental schedule is described in Fig. 2A. (B) Statistical analysis of MFI of VCAM1 in the indicated cell subsets (n=6). (C) Vcam1 mRNA expression in BMECs relative to Actb (n=8). The experimental schedule is described in Fig. 4. (D) MFI of VCAM1 in BMECs from mice with acute hemorrhagic and hemolytic anemia (Bleeding, $\mathrm{n}=6$; PHZ, $\mathrm{n}=5)$.

one that is mediated by cannabinoid receptor 2 , a $G_{a i}$ protein-coupled receptor required for $\mathrm{B}$ cell sinusoidal retention, was affected by erythropoietin (Pereira et al., 2009). Further, we observed a decrease in the expression of Il7 in bone marrow endothelial cells that may suppress B-lymphopoiesis, as previously suggested in MSCs (Nagasawa, 2006; Singbrant et al., 2011).

Erythropoietin specifically released immature and mature B cells and T cells from the bone marrow within 24 $\mathrm{h}$, but not other developing B cells (pre-pro, pro, and pre B cells), neutrophils, and HSCs, which also require VCAM1 and CXCL12 for their retention in the bone marrow (Lapidot and Petit, 2002; Ma et al., 1999; Nagasawa, 2006; Petty et al., 2009; Suratt et al., 2004). This specificity implies that these cells, which are not released by erythropoietin from the bone marrow, mainly retain to other bone marrow stromal cells such as MSCs and not to endothelial cells. In addition, erythropoietin treatment did not recapitu- 
late the emigration of developing B cells from pre-pro B to pre B cells and neutrophils and that is observed in anemic mice, suggesting that the exit of these cells is not directly regulated by the effect of erythropoietin on endothelial cells. Hemorrhagic anemia is often accompanied by the disruption of the blood vasculature and increases the risk of pathogenic organism invasion of the circulation. Therefore, rapid and specific release of lymphocytes from the bone marrow may contribute to an increase in lymphocyte repertoire diversity in the periphery, facilitating efficient responses to antigens.

Anemia can sometimes be a life-threatening condition; therefore, it necessitates a rapid increase in erythrocytes in the periphery. Integrin alpha 4 expression declines at a late stage of erythroblast development, leading to the release of these cells from the bone marrow (Ulyanova et al., 2014). The decrease in VCAM1 expression in endothelial cells induced by erythropoietin may also contribute to the release of erythrocytes in response to acute anemia. Indeed, the number of late stage erythroblasts was significantly decreased in the bone marrow $24 \mathrm{~h}$ after erythropoietin treatment. We also observed increases in the levels of $I l 6$ and $M m p 9$, which are required for the terminal maturation of erythroblasts and proteinase cleavage of stem cell factor in the recovery from myeloablation, respectively (Bernad et al., 1994; Heissig et al., 2002), in bone marrow endothelial cells in response to erythropoietin administration. Thus, in addition to $\mathrm{B}$ cell export to the periphery, bone marrow endothelial cells may also contribute to the promotion of emergency erythropoiesis through the regulation of various hematopoietic factors.

Erythropoietin receptor is expressed in nonhematopoietic cells, such as brain vascular endothelial cells, cardiac myocytes, neuronal cells, and kidneys, and has cytoprotective functions in such cells (Arcasoy, 2008; Ghezzi and Brines, 2004). Erythropoietin receptor expression in bone marrow endothelial cells has previously been suggested from the results of experiments using Epor reporter mice (Singbrant et al., 2011); however, its functional significance in this context remains unclear. Our data demonstrate that Epor expression in bone marrow endothelial cells is significantly higher than that in endothelial cells of other organs. Furthermore, erythropoietin stimulation induced the phosphorylation of STAT5 in bone marrow endothelial cells, strongly suggesting that the receptor is functional. Nevertheless, we cannot rule out the possibility that the observed response was regulated by other signaling pathways or was an indirect effect caused by growth factors and cytokines produced by erythroblasts, as previously suggested (Sathyanarayana et al., 2007; Tordjman et al., 2001).

Accumulating evidence suggests that bone marrow stromal cells regulate the behavior of hematopoietic cells in emergency hematopoiesis as well as during steady state conditions (Boettcher et al., 2014; Day et al., 2015; Shi et al., 2011). Seeing that bone marrow endothelial cells con- stitute sinus vasculature, they may be adapted to sense blood borne cytokines and hormones and to control the egress of hematopoietic cells from the bone marrow. Overall, our findings provide the new insight that bone marrow endothelial cells have the capacity to sense and rapidly respond to elevated circulating erythropoietin in the early phase of anemia, thereby regulating emergency hematopoiesis.

Acknowledgments. The authors thank Drs Y. Omatsu, T. Sugiyama, and T. Nagasawa for technical assistance, and Dr. P. Karagiannis for proofreading. This work was supported by grants from the Japanese Ministry of Education, Culture, Sports, Science and Technology (24590580, 15H01154, 17H05641 to Y.H.; 24111008 to N.M).

\section{References}

Allende, M.L., Tuymetova, G., Lee, B.G., Bonifacino, E., Wu, Y.P., and Proia, R.L. 2010. S1P1 receptor directs the release of immature B cells from bone marrow into blood. J. Exp. Med., 207: 1113-1124.

Arcasoy, M.O. 2008. The non-haematopoietic biological effects of erythropoietin. Br. J. Haematol., 141: 14-31.

Batten, S.J. and Osmond, D.G. 1984. The localization of B lymphocytes in mouse bone marrow: radioautographic studies after in vivo perfusion of radiolabelled anti-IgM antibody. J. Immunol. Methods, 72: 381-399.

Bernad, A., Kopf, M., Kulbacki, R., Weich, N., Koehler, G., and Gutierrez-Ramos, J.C. 1994. Interleukin-6 is required in vivo for the regulation of stem cells and committed progenitors of the hematopoietic system. Immunity, 1: 725-731.

Boettcher, S., Gerosa, R.C., Radpour, R., Bauer, J., Ampenberger, F., Heikenwalder, M., Kopf, M., and Manz, M.G. 2014. Endothelial cells translate pathogen signals into G-CSF-driven emergency granulopoiesis. Blood, 124: 1393-1403.

Cariappa, A., Mazo, I.B., Chase, C., Shi, H.N., Liu, H., Li, Q., Rose, H., Leung, H., Cherayil, B.J., Russell, P., von Andrian, U., and Pillai, S. 2005. Perisinusoidal B cells in the bone marrow participate in Tindependent responses to blood-borne microbes. Immunity, 23: 397407.

Chow, A., Lucas, D., Hidalgo, A., Mendez-Ferrer, S., Hashimoto, D., Scheiermann, C., Battista, M., Leboeuf, M., Prophete, C., van Rooijen, N., Tanaka, M., Merad, M., and Frenette, P.S. 2011. Bone marrow CD169+ macrophages promote the retention of hematopoietic stem and progenitor cells in the mesenchymal stem cell niche. J. Exp. Med., 208: 261-271.

Day, R.B., Bhattacharya, D., Nagasawa, T., and Link, D.C. 2015. Granulocyte colony-stimulating factor reprograms bone marrow stromal cells to actively suppress B lymphopoiesis in mice. Blood, 125: 3114-3117.

Ghezzi, P. and Brines, M. 2004. Erythropoietin as an antiapoptotic, tissueprotective cytokine. Cell Death Differ., 11 Suppl 1: S37-44.

Glodek, A.M., Honczarenko, M., Le, Y., Campbell, J.J., and Silberstein, L.E. 2003. Sustained activation of cell adhesion is a differentially regulated process in B lymphopoiesis. J. Exp. Med., 197: 461-473.

Heissig, B., Hattori, K., Dias, S., Friedrich, M., Ferris, B., Hackett, N.R., Crystal, R.G., Besmer, P., Lyden, D., Moore, M.A., Werb, Z., and Rafii, S. 2002. Recruitment of stem and progenitor cells from the bone marrow niche requires MMP-9 mediated release of kit-ligand. Cell, 109: 625-637.

Jelkmann, W. 1992. Erythropoietin: structure, control of production, and function. Physiol. Rev., 72: 449-489.

Kawamoto, T. 2003. Use of a new adhesive film for the preparation of multi-purpose fresh-frozen sections from hard tissues, whole-animals, insects and plants. Arch. Histol. Cytol., 66: 123-143. 
Koni, P.A., Joshi, S.K., Temann, U.A., Olson, D., Burkly, L., and Flavell, R.A. 2001. Conditional vascular cell adhesion molecule 1 deletion in mice: impaired lymphocyte migration to bone marrow. J. Exp. Med., 193: 741-754.

Krantz, S.B. 1991. Erythropoietin. Blood, 77: 419-434.

Lapidot, T. and Petit, I. 2002. Current understanding of stem cell mobilization: the roles of chemokines, proteolytic enzymes, adhesion molecules, cytokines, and stromal cells. Exp. Hematol., 30: 973-981.

Leuker, C.E., Labow, M., Muller, W., and Wagner, N. 2001. Neonatally induced inactivation of the vascular cell adhesion molecule 1 gene impairs $\mathrm{B}$ cell localization and $\mathrm{T}$ cell-dependent humoral immune response. J. Exp. Med., 193: 755-768.

Levesque, J.P., Takamatsu, Y., Nilsson, S.K., Haylock, D.N., and Simmons, P.J. 2001. Vascular cell adhesion molecule-1 (CD106) is cleaved by neutrophil proteases in the bone marrow following hematopoietic progenitor cell mobilization by granulocyte colony-stimulating factor. Blood, 98: 1289-1297.

Ma, Q., Jones, D., and Springer, T.A. 1999. The chemokine receptor CXCR4 is required for the retention of B lineage and granulocytic precursors within the bone marrow microenvironment. Immunity, 10: 463471.

Martin, C., Burdon, P.C., Bridger, G., Gutierrez-Ramos, J.C., Williams, T.J., and Rankin, S.M. 2003. Chemokines acting via CXCR2 and CXCR4 control the release of neutrophils from the bone marrow and their return following senescence. Immunity, 19: 583-593.

Mazo, I.B., Honczarenko, M., Leung, H., Cavanagh, L.L., Bonasio, R., Weninger, W., Engelke, K., Xia, L., McEver, R.P., Koni, P.A., Silberstein, L.E., and von Andrian, U.H. 2005. Bone marrow is a major reservoir and site of recruitment for central memory CD8+ T cells. Immunity, 22: 259-270.

Mendelson, A. and Frenette, P.S. 2014. Hematopoietic stem cell niche maintenance during homeostasis and regeneration. Nat. Med., 20: 833846.

Morrison, S.J. and Scadden, D.T. 2014. The bone marrow niche for haematopoietic stem cells. Nature, 505: 327-334.

Nagasawa, T. 2006. Microenvironmental niches in the bone marrow required for B-cell development. Nat. Rev. Immunol., 6: 107-116.

Nie, Y., Waite, J., Brewer, F., Sunshine, M.J., Littman, D.R., and Zou, Y.R. 2004. The role of CXCR4 in maintaining peripheral B cell compartments and humoral immunity. J. Exp. Med., 200: 1145-1156.

Osmond, D.G. and Batten, S.J. 1984. Genesis of B lymphocytes in the bone marrow: extravascular and intravascular localization of surface
IgM-bearing cells in mouse bone marrow detected by electronmicroscope radioautography after in vivo perfusion of 125I anti-IgM antibody. Am. J. Anat., 170: 349-365.

Pereira, J.P., An, J., Xu, Y., Huang, Y., and Cyster, J.G. 2009. Cannabinoid receptor 2 mediates the retention of immature B cells in bone marrow sinusoids. Nat. Immunol., 10: 403-411.

Petty, J.M., Lenox, C.C., Weiss, D.J., Poynter, M.E., and Suratt, B.T. 2009. Crosstalk between CXCR4/stromal derived factor-1 and VLA-4/ VCAM-1 pathways regulates neutrophil retention in the bone marrow. J. Immunol., 182: 604-612.

Sapoznikov, A., Pewzner-Jung, Y., Kalchenko, V., Krauthgamer, R., Shachar, I., and Jung, S. 2008. Perivascular clusters of dendritic cells provide critical survival signals to B cells in bone marrow niches. Nat. Immunol., 9: 388-395.

Sathyanarayana, P., Menon, M.P., Bogacheva, O., Bogachev, O., Niss, K., Kapelle, W.S., Houde, E., Fang, J., and Wojchowski, D.M. 2007. Erythropoietin modulation of podocalyxin and a proposed erythroblast niche. Blood, 110: 509-518.

Shi, C., Jia, T., Mendez-Ferrer, S., Hohl, T.M., Serbina, N.V., Lipuma, L., Leiner, I., Li, M.O., Frenette, P.S., and Pamer, E.G. 2011. Bone marrow mesenchymal stem and progenitor cells induce monocyte emigration in response to circulating toll-like receptor ligands. Immunity, 34: 590601.

Singbrant, S., Russell, M.R., Jovic, T., Liddicoat, B., Izon, D.J., Purton, L.E., Sims, N.A., Martin, T.J., Sankaran, V.G., and Walkley, C.R. 2011. Erythropoietin couples erythropoiesis, B-lymphopoiesis, and bone homeostasis within the bone marrow microenvironment. Blood, 117: $5631-5642$.

Suratt, B.T., Petty, J.M., Young, S.K., Malcolm, K.C., Lieber, J.G., Nick, J.A., Gonzalo, J.A., Henson, P.M., and Worthen, G.S. 2004. Role of the CXCR4/SDF-1 chemokine axis in circulating neutrophil homeostasis. Blood, 104: 565-571.

Tordjman, R., Delaire, S., Plouet, J., Ting, S., Gaulard, P., Fichelson, S., Romeo, P.H., and Lemarchandel, V. 2001. Erythroblasts are a source of angiogenic factors. Blood, 97: 1968-1974.

Ulyanova, T., Padilla, S.M., and Papayannopoulou, T. 2014. Stagespecific functional roles of integrins in murine erythropoiesis. Exp. Hematol., 42: 404-409 e404.

(Received for publication, August 28, 2017, accepted, October 8, 2017 and published online, October 25, 2017) 